\title{
Analytical Description of Digital Circles
}

\author{
Eric Andres ${ }^{1}$ and Tristan Roussillon ${ }^{2}$ \\ 1 Laboratoire XLIM, SIC Department, \\ University of Poitiers, BP30179, UMR CNRS 6712, \\ 86962 Futuroscope Chasseneuil Cedex, France \\ andres@sic.univ-poitiers.fr \\ 2 Université de Lyon, \\ Université Lyon 2, LIRIS, UMR CNRS 5205, F-69676, France \\ tristan.roussillon@liris.cnrs.fr
}

\begin{abstract}
In this paper we propose an analytical description of different kinds of digital circles that appear in the literature and especially in digital circle recognition algorithms.
\end{abstract}

\section{Introduction}

Digital primitive recognition is an important topic for the digital geometry and pattern recognition communities. Two of the most basic primitives have been intensively studied, the digital straight line and the digital circle, and many different recognition algorithms have been proposed. One of the key elements in recognizing a digital primitive is actually knowing what object is recognized. This is not as obvious at it seems. Firstly, there are many ways of defining a digital primitive for a given Euclidean one. This means that depending on the considered definition, a same set of pixels can be recognized as a digital primitive or not. Most recognition algorithms provide parameters of the Euclidean primitive and the corresponding digital primitive is implicit. This makes any comparison between different algorithms hard because different sets are recognized. Secondly, there is the actual problem of the definition of a digital primitive. A digital primitive that is only defined as the result of an algorithm or implicitly by a set of properties does not easily allow a global mathematical description.

An analytical description of a digital primitive is interesting for several reasons: it provides a simple way of verifying whether a point or a set of points belongs to the primitive or not, it often provides generalizations that cannot be easily derived from an algorithm. For instance, the Bresenham circle has been defined for integer coordinate centers and integer radii as the result of a digitization algorithm. As we will see, its analytical description straightforwardly extends the previous definition to non integer centers and radii. Extensions to higher dimensions are also possible. Although we don't consider that in this paper, it is one of the perspectives of this paper.

J-P. Reveillès 23] proposed an analytical description for the digital straight lines in 1991. Most, if not all, the digital straight segment recognition algorithms recognize a connected subset of a kind or another of Reveillès digital straight 
lines. In the different digital circle recognition algorithms, there are various digital circle definitions proposed without any analytical characterization. In this paper, we propose analytical definitions of most digital circles, i.e. each digital circle is defined as the solution of a system of analytical inequalities.

After a short recall on digital analytical models and the analytical description of the Andres circle, we propose an analytical description of the supercover, standard and naïve circles. In the same way, we propose an analytical description of the digital circles defined as the boundary of the Gauss digitization of Euclidean circles. We end the paper with a discussion.

\section{Digital Analytical Circle Description}

In this section, we propose analytical descriptions for several definitions that appear in digital circle recognition algorithms.

\subsection{Recall on Digital Analytical Models}

We consider here digital analytical models based on a distance $d$. Let us consider a Euclidean object $E$. The digitization $D_{d}(E)$ of $E$ according to the digital analytical model associated to $d$ is defined by:

$$
D_{d}(E)=\left\{p \in \mathbb{Z}^{2} \mid d(p, E) \leq \frac{1}{2}\right\} .
$$

This global definition is particularly interesting because several digitization models can be derived with respect to the classical distances such as the Manhattan distance $d_{1}$, the Euclidean distance $d_{2}$ or the Tchebychev distance $d_{\infty}$. There are also some basic properties that are very useful when constructing digital objects such as, for $E, F$ two Euclidean objects, $D_{d}(E \cup F)=D_{d}(E) \cup D_{d}(F)$ (see [5] for more details on the supercover analytical model).

There is an equivalent definition that involves a structuring element: the unit sphere $B_{d}(1)$ of diameter one for the distance $d$. The morphological definition can be written as follow:

$$
D_{d}(E)=\left(E \oplus B_{d}(1)\right) \bigcap \mathbb{Z}^{2}
$$

where $A \oplus B=\{a+b, a \in A, b \in B\}$ is the Minkowski sum.

The Euclidean region $E \oplus B_{d}(1)$ is called the offset region. The Pythagorean model is based on the $d_{2}$ distance, the supercover model is based on the $d_{\infty}$ distance and the naive model on the $d_{1}$ distance. These models respectively define the Andres, supercover and closed naive digital circles.

In the following subsections, we focus on the analytical models of the Euclidean circle of center $\left(x_{o}, y_{o}\right) \in \mathbb{R}^{2}$ and radius $R \in \mathbb{R}^{+}$, denoted by $\mathcal{C}\left(x_{o}, y_{o}, R\right)$, and we derive analytical definitions of the above-mentioned digital circles from their morphological definitions. 


\section{$2.2 \quad$ Andres Circle}

The Andres circle, which is based on the Euclidean distance, has been proposed in all dimensions by Andres [6. In two dimensions, it is defined as follows: $(x, y) \in \mathbb{Z}^{2}$ belongs to the Andres circle of center $\left(x_{o}, y_{o}\right)$ and radius $R$ if and only if:

$$
\left(R-\frac{1}{2}\right)^{2} \leq\left(x-x_{o}\right)^{2}+\left(y-y_{o}\right)^{2}<\left(R+\frac{1}{2}\right)^{2}
$$

Note that in this definition, $x_{o}, y_{o}$ and $R$ are not integers. If we consider a closed definition with $\leq$ on both inequalities for the Andres circle then it is easy to see that it is associated to the distance $d_{2}$ :

$$
\left(\mathcal{C} \oplus B_{2}(1)\right) \bigcap \mathbb{Z}^{2}
$$

The recognition of Andres circles can be solved by annulus fitting, which is a problem that has been extensively studied by the computational geometry community [1]. More recently, people of the digital geometry community have also considered the problem of annulus fitting in the case of Andres circles corrupted by noise 28 .

\section{$2.3 \quad$ Supercover Circles}

The supercover model is based on the $d_{\infty}$ distance. In two dimensions, the corresponding structuring element is the unit square $B_{\infty}(1)$, which is the axis-aligned closed unit square, i.e. $B_{\infty}(1)=\left\{(x, y) \in \mathbb{R}^{2} \mid \max (|x|,|y|) \leq \frac{1}{2}\right\}$.

The supercover model is very well adapted for linear objects and every linear object can be described analytically in this model [5]. However, it can also be applied on pieces of $\mathcal{C}^{2}$ curves where the slope of the tangent monotonously increases or decreases like in circle quadrants. For instance, let us consider the circular arc $\mathcal{A}$ of the circle $\mathcal{C}(0,0, R)$ between the angles 0 to $\frac{\pi}{2}$. The offset region $\mathcal{A} \oplus B_{\infty}(1)$ is the union between the closed unit squares centered on $(0, R)$ and $(R, 0)$ at both ends of $\mathcal{A}$, and the region bounded by the translation of $\mathcal{A}$ by the vector $\left(\frac{1}{2}, \frac{1}{2}\right)$, the translation of $\mathcal{A}$ by the vector $\left(-\frac{1}{2},-\frac{1}{2}\right)$, the straight segment joining $\left(-\frac{1}{2}, R-\frac{1}{2}\right)$ and $\left(\frac{1}{2}, R+\frac{1}{2}\right)$, the straight segment joining $\left(R-\frac{1}{2},-\frac{1}{2}\right)$ and $\left(R+\frac{1}{2}, \frac{1}{2}\right)$ (fig. 1 a).

Due to symmetries, the offset region of the three other quadrants is defined in the same way so that each point of $\mathcal{C} \oplus B_{\infty}(1)$ either lies in one of the four closed unit squares centered in $(0, R),(R, 0),(0,-R),(-R, 0)$ or belongs to one of the four disks of radius $R$ and center $\left(-\frac{1}{2},-\frac{1}{2}\right),\left(\frac{1}{2}, \frac{1}{2}\right),\left(-\frac{1}{2}, \frac{1}{2}\right),\left(\frac{1}{2},-\frac{1}{2}\right)$, but not to both of them (fig. 1,b).

We can thus derive an analytical description of the supercover circle:

Proposition 1 (Supercover of a circle). A point $(x, y) \in \mathbb{Z}^{2}$ belongs to the supercover circle $\mathbb{C}_{\infty}\left(x_{o}, y_{o}, R\right)=\left(\left(\mathcal{C} \oplus B_{\infty}(1)\right) \cap \mathbb{Z}^{2}\right)$, if and only if: 


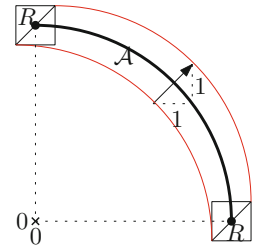

(a)

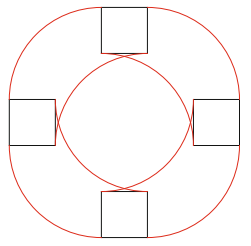

(b)

Fig. 1. Construction of the offset region used to define the supercover of a circle

$$
\begin{array}{r}
\left|y-y_{o}\right| \leq \frac{1}{2} \text { and }\left|\left(\left|x-x_{o}\right|-R\right)\right| \leq \frac{1}{2} \\
\text { or } \\
\left|x-x_{o}\right| \leq \frac{1}{2} \text { and }\left|\left(\left|y-y_{o}\right|-R\right)\right| \leq \frac{1}{2} \\
\text { or } \\
R^{2}-\frac{1}{2}-\left(\left|x-x_{o}\right|+\left|y-y_{o}\right|\right) \leq\left(x-x_{o}\right)^{2}+\left(y-y_{o}\right)^{2} \leq \\
R^{2}-\frac{1}{2}+\left(\left|x-x_{o}\right|+\left|y-y_{o}\right|\right)
\end{array}
$$

Proof. The first part of the analytical description corresponds to the squares at the cardinal points and is obvious. We get the last part of the analytical description by developing and applying axial symmetries to the equations for the first quadrant $\left(x-x_{o}-\frac{1}{2}\right)^{2}+\left(y-y_{o}-\frac{1}{2}\right)^{2} \geq R^{2}$ and $\left(x-x_{o}+\frac{1}{2}\right)^{2}+\left(y-y_{o}+\frac{1}{2}\right)^{2} \leq$ $R^{2}$.

The supercover of $\mathbb{C}_{\infty}(0,0,5)$ and $\mathbb{C}_{\infty}\left(\frac{1}{2}, \frac{1}{2}, 5\right)$ are respectively depicted in fig. 2, a and $b$.

Note that Lincke proposed another interpretation of this result based on mathematical morphology operations [17][fig. 4].

Note in addition that Nakamura and Aizawa, based on a cellular scheme, defined a digital disk 20] that is actually a supercover disk. The outer border of their digital disk is thus also the outer border of a supercover circle.

\subsection{Standard Analytical Circles}

The standard model has been defined only for linear primitives in 44. In the supercover model, when the Euclidean object passes through a point $p$ composed only of half-integer coordinates, there is a bubble, i.e. four digital points that are the vertices of the axis-aligned closed unit square centered on $p$. For instance, the supercover of the point $\left(\frac{1}{2}, \frac{1}{2}\right)$ is composed of the digital points $\{(0,0) ;(0,1) ;(1,0) ;(1,1)\}$. Similarly, in fig. 2 .b, the points $\left(5+\frac{1}{2}, \frac{1}{2}\right),\left(4+\frac{1}{2}, 3+\frac{1}{2}\right)$, $\left(3+\frac{1}{2}, 4+\frac{1}{2}\right),\left(\frac{1}{2}, 5+\frac{1}{2}\right)$ (only for the first quadrant) are lying on $\mathcal{C}\left(\frac{1}{2}, \frac{1}{2}, 5\right)$ and are thus center of bubbles.

There can be two different definitions of standard circles depending if we remove the outer or inner border of the offset region $\mathcal{C} \oplus B_{\infty}(1)$. The analytical 


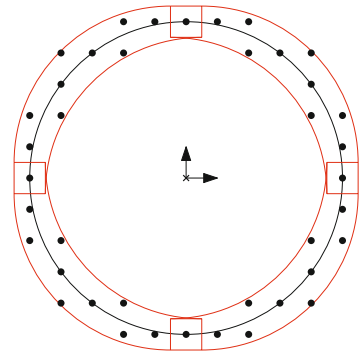

(a)

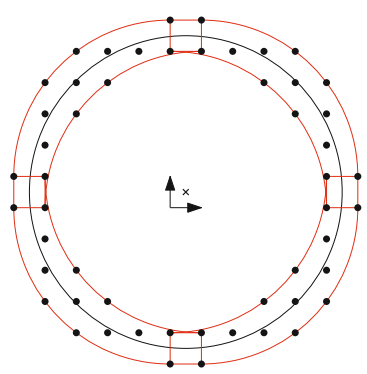

(b)

Fig. 2. The supercover of $\mathbb{C}_{\infty}(0,0,5)$ (a) and $\mathbb{C}_{\infty}\left(\frac{1}{2}, \frac{1}{2}, 5\right)$ (b) is depicted with black disks

description of the outer standard circle $\mathbb{C}_{\infty}^{+}\left(x_{o}, y_{o}, R\right)$ is obtained by replacing $\left|x-x_{o}\right| \leq R+\frac{1}{2}$ by $\left|x-x_{o}\right|<R+\frac{1}{2},\left|y-y_{o}\right| \leq R+\frac{1}{2}$ by $\left|y-y_{o}\right|<R+\frac{1}{2}$ and $\left(x-x_{o}\right)^{2}+\left(y-y_{o}\right)^{2} \leq R^{2}-\frac{1}{2}+\left(\left|x-x_{o}\right|+\left|y-y_{o}\right|\right)$ by $\left(x-x_{o}\right)^{2}+\left(y-y_{o}\right)^{2}<R^{2}-\frac{1}{2}+$ $\left(\left|x-x_{o}\right|+\left|y-y_{o}\right|\right)$ in the analytical description of the supercover circle (the outer border is removed). Similarly, the analytical description of the inner standard circle $\mathbb{C}_{\infty}^{-}\left(x_{o}, y_{o}, R\right)$ is obtained by simply replacing $R^{2}-\frac{1}{2}-\left(\left|x-x_{o}\right|+\left|y-y_{o}\right|\right) \leq$ $\left(x-x_{o}\right)^{2}+\left(y-y_{o}\right)^{2}$ by $R^{2}-\frac{1}{2}-\left(\left|x-x_{o}\right|+\left|y-y_{o}\right|\right)<\left(x-x_{o}\right)^{2}+\left(y-y_{o}\right)^{2}$ in the supercover circle definition (the inner border is removed). The inner standard circle $\mathbb{C}_{\infty}^{-}\left(\frac{1}{2}, \frac{1}{2}, 5\right)$ is depicted in fig. 3. a.

Most of the bubbles are removed in the standard model (compare for instance fig. 2.b and fig. 3]a). However, neither the outer nor the inner standard circles are always simply 4-connected because it may remain a bubble around one of the four cardinal points (like in fig. 3.a). The inner standard circle is however interesting

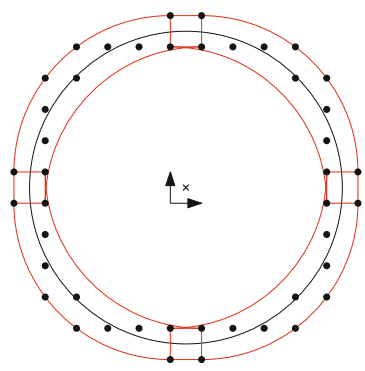

(a)

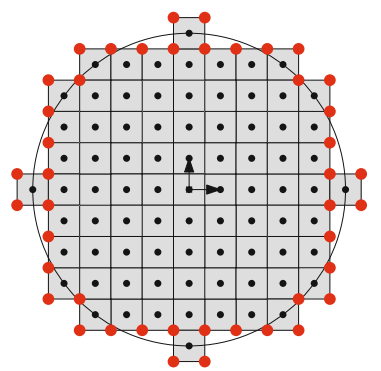

(b)

Fig. 3. The inner standard analytical circle $\mathbb{C}_{\infty}^{-}\left(\frac{1}{2}, \frac{1}{2}, 5\right)$ is depicted in (a) with black disks. The Kovalevsky circle $\mathbb{K}(0,0,5)$ is depicted in (b) with big disks along the boundary of the gray area. Note that $\mathbb{C}_{\infty}^{-}\left(\frac{1}{2}, \frac{1}{2}, 5\right)$ and $\mathbb{K}(0,0,5)$ are similar objects up to a translation 
because it corresponds to the Kovalevsky circle [15], denoted by $\mathbb{K}\left(x_{o}, y_{o}, R\right)$ and defined as the set of points of half-integer coordinates $(x, y) \in\left(\mathbb{Z}+\frac{1}{2}\right) \times\left(\mathbb{Z}+\frac{1}{2}\right)$ that belongs to the boundary of the dilatation of the Gauss digitization of the interior of of $\mathcal{C}\left(x_{o}, y_{o}, R\right)$ by $B_{\infty}(1)$, i.e. the boundary of $\left\{(i, j) \in \mathbb{Z}^{2} \mid\left(i-x_{o}\right)^{2}+\right.$ $\left.\left(j-y_{o}\right)^{2} \leq R^{2}\right\} \oplus B_{\infty}(1)$.

In fig. 3. b, the Gauss digitization of $\mathcal{C}(0,0,5)$ is depicted with black disks. The gray area is its dilatation by $B_{\infty}(1)$. Kovalevsky circle is depicted with red disks lying along the boundary of the gray area.

Proposition 2. The point $(x, y) \in \mathbb{Z}^{2}$ belong to $\mathbb{C}_{\infty}^{-}\left(x_{o}+\frac{1}{2}, y_{o}+\frac{1}{2}, R\right)$ if and only if the point $\left(x-\frac{1}{2}, y-\frac{1}{2}\right)$ belongs to $\mathbb{K}\left(x_{o}, y_{o}, R\right)$.

Proof. Firstly, we will prove that if the point $\left(x-\frac{1}{2}, y-\frac{1}{2}\right)$ belong to $\mathbb{K}\left(x_{o}, y_{o}, R\right)$, then the point $(x, y)$ belongs to $\mathbb{C}_{\infty}^{-}\left(x_{o}+\frac{1}{2}, y_{o}+\frac{1}{2}, R\right)$ (i) and secondly, we will prove that if the point $\left(x-\frac{1}{2}, y-\frac{1}{2}\right)$ does not belong to $\mathbb{K}\left(x_{o}, y_{o}, R\right)$, then $(x, y)$ does not belong to $\mathbb{C}_{\infty}^{-}\left(x_{o}+\frac{1}{2}, y_{o}+\frac{1}{2}, R\right)$.

Let us shortly denote by $\mathcal{C}$ the circle of center $\left(x_{o}, y_{o}\right) \in \mathbb{R}^{2}$ and radius $R \in \mathbb{R}^{+}$. For all points $p(x, y) \in\left(\mathbb{Z}+\frac{1}{2}\right) \times\left(\mathbb{Z}+\frac{1}{2}\right)$, the four vertices of $p \oplus B_{\infty}(1)$ are points of $\mathbb{Z}^{2}$. Those that are enclosed by $\mathcal{C}$ or lie on $\mathcal{C}$ are referred as foreground points, the others are referred as background points.

(i) For all points $p\left(x-\frac{1}{2}, y-\frac{1}{2}\right) \in \mathbb{K}\left(x_{o}, y_{o}, R\right)$, at least one vertex of $p \oplus B_{\infty}(1)$ is a foreground point and at least one is a background point, because $p$ is assumed to belong to $\mathbb{K}\left(x_{o}, y_{o}, R\right)$. As a consequence, $\mathcal{C}$ must intersect $p \oplus B_{\infty}(1)$ and by duality $\mathcal{C} \oplus B_{\infty}(1)$ contains $p$. Note that since $\mathcal{C}$ cannot pass through any background point, $p$ does not belong to the inner border of $\mathcal{C} \oplus B_{\infty}$ (1) (if any), but is either in its interior or is lying on the outer border. Since this membership is preserved under translation, $(x, y)$ belongs to $\mathbb{C}_{\infty}^{-}\left(x_{o}+\frac{1}{2}, y_{o}+\frac{1}{2}, R\right)$.

(ii) For all points $p\left(x-\frac{1}{2}, y-\frac{1}{2}\right) \notin \mathbb{K}\left(x_{o}, y_{o}, R\right)$, the four vertices of $p \oplus B_{\infty}(1)$ are either all foreground points or all background points. In the last case, $\mathcal{C}$ does not intersect $p \oplus B_{\infty}(1)$ and by duality $\mathcal{C} \oplus B_{\infty}(1)$ does not contain $p$ and we are done. In the first case, $\mathcal{C}$ may pass through one of the foreground points and $p$ may thus lie in the inner border of $\mathcal{C} \oplus B_{\infty}(1)$ (which always exists in this case). Since the inner border of the offset region is removed in the standard model and since the incident relations are preserved under translation, $(x, y)$ does not belong to $\mathbb{C}_{\infty}^{-}\left(x_{o}+\frac{1}{2}, y_{o}+\frac{1}{2}, R\right)$.

Due to this proposition, the recognition algorithm of Kovalevsky [15] provides a way of recognizing inner standard circles.

\subsection{Closed Naïve and Naïve Analytical Circles}

The naïve model, introduced in $\left[3\right.$, is based on the $d_{1}$ distance. In two dimensions, the corresponding structuring element is the unit square $B_{1}(1)$, which is a square of side size $\frac{\sqrt{2}}{2}$ and with a $45^{\circ}$ rotation compared to $B_{\infty}(1)$, i.e. $B_{1}(1)=\left\{(x, y) \in \mathbb{R}^{2} \mid(|x|+|y|) \leq \frac{1}{2}\right\}$. 
The analytical description of the closed naïve circle is therefore very similar to the one of the supercover circle (compare the offset region defining a closed naïve circle in fig. 4, a and the one defining a supercover circle in fig. 1, b).

Proposition 3 (Closed naïve circle). A point $(x, y) \in \mathbb{Z}^{2}$ belongs to the closed naïve circle $\mathbb{C}_{1}\left(x_{o}, y_{o}, R\right)=\left(\left(\mathcal{C} \oplus B_{1}(1)\right) \cap \mathbb{Z}^{2}\right)$, if and only if:

$$
\begin{gathered}
\left|(x-y)-\left(x_{o}-y_{o}\right)\right| \leq \frac{1}{2} \text { and }|| x+y-\left(x_{o}+y_{o}\right)|-R \sqrt{2}| \leq \frac{1}{2} \\
\left|(x+y)-\left(x_{o}+y_{o}\right)\right| \leq \frac{1}{2} \text { and } \underset{\text { or }}{|| x-y-\left(x_{o}-y_{o}\right)|-R \sqrt{2}| \leq \frac{1}{2}} \\
R^{2}-\frac{1}{4}-\max \left(\left|x-x_{o}\right|,\left|y-y_{o}\right|\right) \leq\left(x-x_{o}\right)^{2}+\left(y-y_{o}\right)^{2} \\
\leq R^{2}-\frac{1}{4}+\max \left(\left|x-x_{o}\right|,\left|y-y_{o}\right|\right)
\end{gathered}
$$

Proof. The proof of this proposition is very similar to the proof of proposition 1 . The last part of the equations is obtained by developing and applying the corresponding symmetries to $\left(x-x_{o}-\frac{1}{2}\right)^{2}+\left(y-y_{o}\right)^{2} \geq R^{2}$ and $\left(x-x_{o}+\frac{1}{2}\right)^{2}+\left(y-y_{o}\right)^{2} \leq$ $R^{2}$.

Similarly to the two definitions of standard circles, it is possible to define an inner naïve circle, denoted by $\mathbb{C}_{1}^{-}\left(x_{o}, y_{o}, R\right)$, and an outer naïve circle, denoted by $\mathbb{C}_{1}^{+}\left(x_{o}, y_{o}, R\right)$, by removing the inner or outer border of the of the offset region $\mathcal{C} \oplus B_{1}(1)$. Due to this convention, when $\mathcal{C}$ crosses a point of coordinates $\left(x+\frac{1}{2}, y\right)$ in the first octant, $\mathbb{C}_{1}^{-}$does not contain $(x, y)$, whereas $\mathbb{C}_{1}^{+}$does not contain $(x+1, y)$. For instance, in fig. 4, b, we can see the closed naïve circle $\mathbb{C}_{1}\left(\frac{1}{4}, \frac{1}{4}, \frac{7}{2}\right)$. The Euclidean circle $\mathcal{C}$ crosses the points of coordinates $\left(-1,3+\frac{1}{2}\right)$, $\left(3+\frac{1}{2},-1\right),\left(-2,-2-\frac{1}{2}\right)$ and $\left(-2-\frac{1}{2},-2\right)$ (clockwise from the top-left point), which implies that the points $(-2,3),(0,4),(4,0),(3,-2),(-2,-3),(-2,-2)$ and $(-3,-2)$ have three 8-neighbors. However, $\mathbb{C}_{1}^{-}\left(\frac{1}{4}, \frac{1}{4}, \frac{7}{2}\right)\left(\right.$ resp. $\left.\mathbb{C}_{1}^{+}\left(\frac{1}{4}, \frac{1}{4}, \frac{7}{2}\right)\right)$ is simply 8 -connected because it does not contain the points $(-1,3),(3,-1)$, $(-2,-2)$ (resp. $(-1,4),(4,-1),(-2,-3)$ and $(-3,-2))$.

An inner or outer naïve circle may nonetheless not always be simply 8connected because sharp corners may occur at octant boundaries (fig. 4.c). Exactly the same thing happens for Bresenham circles [7] as it is well known (see for instance [18] [section 5]) because of the following proposition:

Proposition 4 (Bresenham Circle). Bresenham circle is a closed, inner and outer naïve circle.

Proof. Let us assume that $x_{o}, y_{o}, R$ are integers. A Bresenham circle is a closed 8 -connected digital curve that is the digitization of $\mathcal{C}\left(x_{o}, y_{o}, R\right)$. Moreover, its points are the closest ones to $\mathcal{C}\left(x_{o}, y_{o}, R\right)$ [16. As such it corresponds to the naïve digitization model.

Moreover no point $\left(x \pm \frac{1}{2}, y\right)$ or $\left(x, y \pm \frac{1}{2}\right)$, with $(x, y) \in \mathbb{Z}^{2}$, belongs to a Euclidean circle that has a center of integer coordinates and an integer radius. 


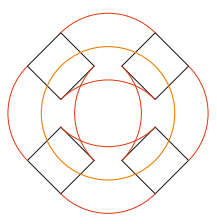

(a)

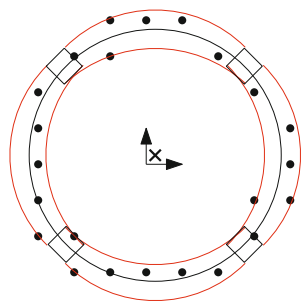

(b)

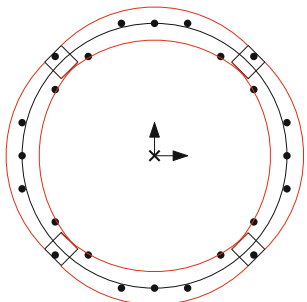

(c)

Fig. 4. Offset region defining a closed naïve circle (a). A closed naïve circle $\mathbb{C}_{1}\left(\frac{1}{4}, \frac{1}{4}, \frac{7}{2}\right)$ (b). Bresenham circle of radius 4 that is not simply 8-connected (c).

The closed, inner and outer naïve models of such circles are thus identical and corresponds to their Bresenham digitization.

This proposition shows that the analytical description we propose for naïve circles is an extension of the Bresenham circles to arbitrary centers and radii. If we consider that the natural extension of the Bresenham circle corresponds to the circles that are simply 8-connected except around octant boundaries, then the outer naïve circle is the best choice. This corresponds to the definition proposed by Pham 22] (see [2]). Note that there is a slight mistake in the starting point in Pham's generation algorithm. This type of mistake is difficult to spot with a generation algorithm. One advantage of an analytical definition is that it can be used to test such algorithms.

Several papers have dealt with the problem of recognizing naïve circles: the paper of Pham [22] in 1992 for outer naïve circles and the papers of Sauer [24] in 1993 and Damaschke 9] in 1995 for Bresenham circles.

\section{Gauss Type Digitized Circles}

In this section, we propose two analytical definitions that do not directly rely on the global model presented in section 2.1 but that are very similar.

Definition 1 ( $d_{1}$-Gauss circle). A point $(x, y)$ belongs to the $d_{1}$-Gauss circle $\mathbb{G}_{\infty}\left(x_{o}, y_{o}, R\right)$ if and only if:

$$
R^{2}-2 \max \left(\left|x-x_{o}\right|,\left|y-y_{o}\right|\right)-1<\left(x-x_{o}\right)^{2}+\left(y-y_{o}\right)^{2} \leq R^{2}
$$

Definition 2 ( $d_{\infty}$-Gauss circle). A point $(x, y)$ belongs to the $d_{\infty}$-Gauss circle $\mathbb{G}_{1}\left(x_{o}, y_{o}, R\right)$ if and only if:

$$
R^{2}-2\left(\left|x-x_{o}\right|+\left|y-y_{o}\right|\right)-1<\left(x-x_{o}\right)^{2}+\left(y-y_{o}\right)^{2} \leq R^{2}
$$

It is easy to sketch the region where the points of $\mathbb{G}_{1}\left(x_{o}, y_{0}, R\right)$ or $\mathbb{G}_{\infty}$ $\left(x_{o}, y_{o}, R\right)$ lie (it is bounded by circular arcs in fig. 55). 


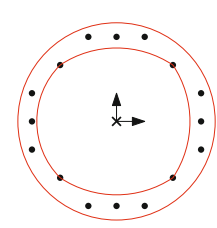

(a)

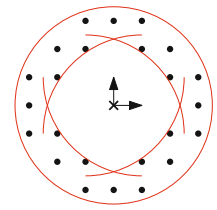

(b)

Fig. 5. Illustration of $\mathbb{G}_{1}(0,0,3.5)$ in (a) and $\mathbb{G}_{\infty}(0,0,3.5)$ in (b)

These digital circles actually correspond to the boundary of the Gauss digitization of $\mathcal{C}$. More precisely:

Proposition 5. The $d_{1}$-Gauss (resp. $d_{\infty}$-Gauss) circle $\mathbb{G}_{1}\left(x_{o}, y_{o}, R\right)$ (resp. $\left.\mathbb{G}_{\infty}\left(x_{o}, y_{o}, R\right)\right)$ is the set of points of the Gauss digitization of the interior of $\mathcal{C}\left(x_{o}, y_{o}, R\right)$, denoted by $D_{G}(\mathcal{C})$, such that their 4-(resp. 8-)neighborhood is not totally included in $D_{G}(\mathcal{C})$.

Proof. We will focus on $\mathbb{G}_{1}\left(x_{o}, y_{o}, R\right)$ because the proof about $\mathbb{G}_{\infty}\left(x_{o}, y_{o}, R\right)$ is the same. Firstly (i), we will prove that if $p \in \mathbb{G}_{1}\left(x_{o}, y_{0}, R\right)$, then the 4neighborhood of $p$ is not totally included in $D_{G}(\mathcal{C})$ and secondly (ii), we will prove that if $p \notin \mathbb{G}_{1}\left(x_{o}, y_{o}, R\right)$, then either the 4-neighborhood of $p$ is totally included in $D_{G}(\mathcal{C})$ or not included at all.

(i) For all $p(x, y) \in \mathbb{G}_{1}\left(x_{o}, y_{o}, R\right),\left(x-x_{o}\right)^{2}+\left(y-y_{o}\right)^{2} \leq R^{2}$ and therefore $p \in$ $D_{G}(\mathcal{C})$. Let us now assume that $p$ lies in the first octant, i.e. $\left(x-x_{o}\right)>\left(y-y_{o}\right) \geq$ 0 . On the one hand $\left(x+1-x_{o}\right)^{2}+\left(y-y_{o}\right)^{2}=\left(x-x_{o}\right)^{2}+\left(y-y_{o}\right)^{2}+2\left(x-x_{o}\right)+1$. On the other hand $\left(x-x_{o}\right)^{2}+\left(y-y_{o}\right)^{2}>R^{2}-2\left(x-x_{o}\right)-1$ due to definition 1] As a result, $\left(x+1-x_{o}\right)^{2}+\left(y-y_{o}\right)^{2}>R^{2}$, i.e. $(x+1, y) \notin D_{G}(\mathcal{C})$. Due to symmetries, we can conclude that the 4-neighborhood of $p$ is not totally included in $D_{G}(\mathcal{C})$ for all $p(x, y) \in \mathbb{G}_{1}\left(x_{o}, y_{o}, R\right)$.

(ii) For all $p(x, y) \notin \mathbb{G}_{1}\left(x_{o}, y_{o}, R\right)$, two cases must be distinguished. If $p \notin$ $D_{G}(\mathcal{C})$, we are done. Otherwise, let us assume that $p$ lies in the first octant, i.e. $\left(x-x_{o}\right)>\left(y-y_{o}\right) \geq 0$. Since $\left(x-x_{o}\right)^{2}+\left(y-y_{o}\right)^{2} \leq R^{2}-2\left(x-x_{o}\right)-1$ in that case, we have $\left(x+1-x_{o}\right)^{2}+\left(y-y_{o}\right)^{2}<R^{2}$, i.e. $(x+1, y) \in D_{G}(\mathcal{C})$. Due to symmetries, we can conclude that the 4-neighborhood of $p$ is totally included in $D_{G}(\mathcal{C})$ in that case and we are done.

The $d_{1}$-Gauss circles, also referred as circles digitized under Kim scheme in

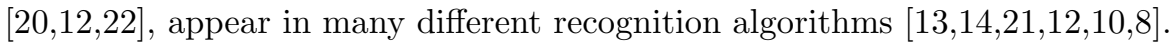

\section{Discussion and Perspectives}

In this paper we have presented analytical inequalities describing the supercover, inner and outer standard, closed naïve, inner and outer naïve, $d_{1}$ - and $d_{\infty}$-Gauss circles. 
Fiorio et. al. 11] proposed an analytical characterization for standard and naïve circles that is very close to the one we are proposing here. They obtained their formula based on differential considerations and thus obtained only the last part of the analytical descriptions. Geometrically, the offset region involved in their approach is only made up with four disks without any square. In addition, the Euclidean circle whose parameters are the same as the ones of the standard or naïve circle is not perfectly centered within the offset region. Therefore, their approach could not be used to characterize existing digital circles (like Kovalevsky circle) as we did in this paper.

Having an analytical characterization has many advantages: it provides a way of verifying if a given set of digital points is a given digital circle or a subset of such a digital circle, it provides a way of verifying the correctiveness of digital circle generation algorithms. Furthermore, our approach leads to a unified framework for digital circle recognition algorithms based on linear programming techniques. The only exception is Andres circles that can be handled through algorithms based on annulus fitting.

Let $\Sigma$ be a set of $n$ digital points. Is $\Sigma$ a given digital circle $\mathbb{C}\left(x_{o}, y_{o}, R\right)$ (where $\mathbb{C} \in\left\{\mathbb{C}_{\infty}, \mathbb{C}_{\infty}^{-}, \mathbb{C}_{\infty}^{+}, \mathbb{C}_{1}, \mathbb{C}_{1}^{-}, \mathbb{C}_{1}^{+}, \mathbb{G}_{\infty}, \mathbb{G}_{1}\right\}$ ) ?

We give below a general scheme in two steps in order to solve the recognition problem using linear programming. This approach is not new and has been used in [9] for Bresenham circles, but we extend it to all the above-mentioned digital circles.

The first step consists in setting a straight segment joining a point $s$ and a point $t$ to each digital point $p \in \Sigma$, such that $\mathcal{C}\left(x_{o}, y_{o}, R\right)$ intersects $[s t]$ if and only if $p$ belongs to $\mathbb{C}\left(x_{o}, y_{o}, R\right)$. This step can be easily performed if the octant (with respect to $\left(x_{o}, y_{o}\right)$ ) where each $p \in \Sigma$ lies is known. We do not provide further details due to lack of space, but the octant of all $p \in \Sigma$ can be deduced from $\Sigma$ in linear time. The straight segments assigned to each digital point of $\mathbb{C}_{\infty}(0,0,5)(\mathrm{a}), \mathbb{C}_{1}(0.5,0.5,3.5)(\mathrm{b})$ and $\mathbb{G}_{1}(0,0,3)(\mathrm{c})$ are depicted in fig. 6. Note that $s$ is not included for $\mathbb{C} \in\left\{\mathbb{C}_{\infty}^{+}, \mathbb{C}_{1}^{+}\right\}$in $[s t]$, whereas $t$ is not included in [st] for $\mathbb{C} \in\left\{\mathbb{C}_{\infty}^{-}, \mathbb{C}_{1}^{-}, \mathbb{G}_{\infty}, \mathbb{G}_{1}\right\}$ (see fig. 6. for instance).

In the second step, the sets of points $s \in S$ and $t \in T$ provide the constraints of a convex program that be translated into a linear one [219] and can then be solved in linear-time using Megiddo prune and search technique [19] or in expected linear-time using Seidel randomized technique [25].

That's why all the recognition algorithms that appear in the literature 13 14 21 12 15 22 24 27/108 are all techniques of solving a unique linear program. The difference lies in the manner of solving the problem.

In 219], a 3D point belonging to the intersection of $2 n$ half-spaces in the parameters space is searched with Megiddo algorithm. In the space that is dual to the parameters space, a plane separating two sets of $n 3 \mathrm{D}$ points is searched using tools coming from computational geometry [10]. In [12 15 22 27/8] a 2D point belonging to the intersection of $n^{2}$ half-planes is searched in the original plane using either brute-force algorithms [15 22 27] or tools coming from computational geometry [128]. 


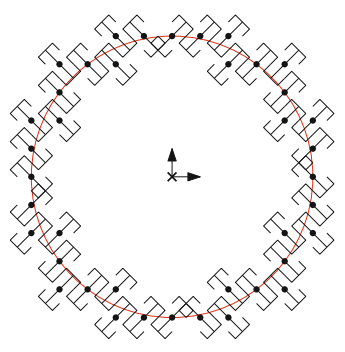

(a)

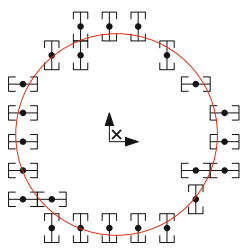

(b)

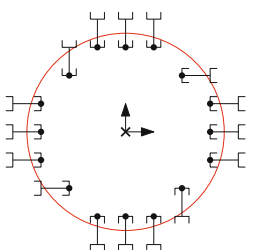

(c)

Fig. 6. Constraints assigned to each digital point of $\mathbb{C}_{\infty}(0,0,5)(\mathrm{a}), \mathbb{C}_{1}(0.5,0.5,3.5)$ (b) and $\mathbb{G}_{1}(0,0,3)$ (c)

One of the main perspective of this paper is of course the extensions that analytical descriptions allow: extension to thick digital circles (by considering structuring elements $B_{d}(k)$ with $k>1$ ) and extension to higher dimensions, which seems possible but not trivial. Another perspective is the extension to more complex algebraic curves [26].

\section{References}

1. Agarwal, P.K., Sharir, M.: Efficient randomized algorithms for some geometric optimization problems. In: Proceedings of the 11th Annual ACM Symposium on Computational Geometry, pp. 326-335 (1995)

2. Andres, E.: Discrete circles, rings and spheres. Computer and Graphics 18(5), 695706 (1994)

3. Andres, E.: Modélisation Analytique Discrète d'Objets Géométriques. Habilitation à diriger des recherches, Université de Poitiers (2000)

4. Andres, E.: Discrete linear objects in dimension n: the standard model. Graphical Models 65(1-3), 92-111 (2003)

5. Andres, E.: The supercover of an $\mathrm{m}$-flat is a discrete analytical object. TCS 406(1-2), 8-14 (2008), http://www.sciencedirect.com/science/article/ B6V1G-4T4J88B-2/2/4101ff67ac5a1fb9c8028c53147a5218, discrete Tomography and Digital Geometry: In memory of Attila Kuba

6. Andres, E., Jacob, M.A.: The discrete analytical hyperspheres. IEEE Transactions on Visualization and Computer Graphics 3(1), 75-86 (1997)

7. Bresenham, J.: A linear algorithm for incremental digital display of circular arcs. Communications of the ACM 20(2), 100-106 (1977)

8. Coeurjolly, D., Gérard, Y., Reveillès, J.P., Tougne, L.: An Elementary Algorithm for Digital Arc Segmentation. Discrete Applied Math. 139(1-3), 31-50 (2004)

9. Damaschke, P.: The Linear Time Recognition of Digital Arcs. Pattern Recognition Letters 16, 543-548 (1995)

10. Efrat, A., Gotsman, C.: Subpixel Image Registration Using Circular Fiducials. Int. Journal of Computational Geometry \& Applications 4(4), 403-422 (1994)

11. Fiorio, C., Jamet, D., Toutant, J.L.: Discrete circles: an arithmetical approach with non-constant thickness. In: Vision Geometry XIV, Electronic Imaging, SPIE, vol. 6066, pp. 60660C.1-60660C.12 (2006) 
12. Fisk, S.: Separating Points Sets by Circles, and the Recognition of Digital Disks. IEEE Transactions on Pattern Analysis and Machine Intelligence 8, 554-556 (1986)

13. Kim, C.E.: Digital Disks. IEEE Transactions on Pattern Analysis and Machine Intelligence 6(3), 372-374 (1984)

14. Kim, C.E., Anderson, T.A.: Digital Disks and a Digital Compactness Measure. In: Annual ACM Symposium on Theory of Computing, pp. 117-124 (1984)

15. Kovalevsky, V.A.: New Definition and Fast Recognition of Digital Straight Segments and Arcs. In: Internation Conference on Pattern Analysis and Machine Intelligence, pp. 31-34 (1990)

16. Kulpa, Z., Doros, M.: Freeman digitization of integer circles minimizes the radial error. Computer Graphics and Image Processing 17(2), 181-184 (1981)

17. Lincke, C., Wüthrich, C.A.: Towards a unified approach between digitization of linear objects and discrete analytical objects. In: WSGG 2000, University of West Bohemia, Plzen Tcheque Republic, pp. 124-131 (2000)

18. McIlroy, M.D.: Best approximate circles on integer grids. ACM Transactions on Graphics 2(4), 237-263 (1983)

19. Megiddo, N.: Linear Programming in Linear Time When the Dimension Is Fixed. SIAM Journal on Computing 31, 114-127 (1984)

20. Nakamura, A., Aizawa, K.: Digital Circles. Computer Vision, Graphics, and Image Processing 26(2), 242-255 (1984)

21. O'Rourke, J., Kosaraju, S.R., Meggido, N.: Computing Circular Separability. Discrete and Computational Geometry 1, 105-113 (1986)

22. Pham, S.: Digital Circles With Non-Lattice Point Centers. The Visual Computer 9, 1-24 (1992)

23. Reveillès, J.P.: Géométrie Discrète, calculs en nombres entiers et algorithmique. Thèse d'etat, Université Louis Pasteur (1991)

24. Sauer, P.: On the Recognition of Digital Circles in Linear Time. Computational Geometry 2(5), 287-302 (1993)

25. Seidel, R.: Small-dimensional linear programming and convex hulls made easy. Discrete and Computational Geometry 6(1), 423-434 (1991)

26. Tajine, M., Ronse, C.: Hausdorff discretizations of algebraic sets and diophantine sets. In: Nyström, I., Sanniti di Baja, G., Borgefors, G. (eds.) DGCI 2000. LNCS, vol. 1953, pp. 99-110. Springer, Heidelberg (2000), http://portal.acm. org/citation. $\mathrm{cfm}$ ? id $=648319.754197$

27. Worring, M., Smeulders, A.W.M.: Digitized Circular Arcs: Characterization and Parameter Estimation. IEEE Transactions on Pattern Analysis and Machine Intelligence 17(6), 554-556 (1995)

28. Zrour, R., Largeteau-Skapin, G., Andres, E.: Optimal Consensus set for Annulus Fitting. In: Debled-Rennesson, I., et al. (eds.) DGCI 2011. LNCS, vol. 6607, pp. 238-249. Springer, Heidelberg (2011) 